\title{
Interdependent networks with correlated degrees of mutually dependent nodes
}

\author{
Sergey V. Buldyrev ${ }^{1}$, Nathaniel Shere ${ }^{1}$, and Gabriel A. Cwilich ${ }^{1}$ \\ ${ }^{1}$ Department of Physics, Yeshiva University, \\ 500 West 185th Street, New York, \\ New York 10033, USA
}

\section{(Dated: Revised manuscript: October 15, 2018)}

\begin{abstract}
We study a problem of failure of two interdependent networks in the case of correlated degrees of mutually dependent nodes. We assume that both networks (A and B) have the same number of nodes $N$ connected by the bidirectional dependency links establishing a one-to-one correspondence between the nodes of the two networks in a such a way that the mutually dependent nodes have the same number of connectivity links, i.e. their degrees coincide. This implies that both networks have the same degree distribution $P(k)$. We call such networks correspondently coupled networks $(\mathrm{CCN})$. We assume that the nodes in each network are randomly connected. We define the mutually connected clusters and the mutual giant component as in earlier works on randomly coupled interdependent networks and assume that only the nodes which belong to the mutual giant component remain functional. We assume that initially a $1-p$ fraction of nodes are randomly removed due to an attack or failure and find analytically, for an arbitrary $P(k)$, the fraction of nodes $\mu(p)$ which belong to the mutual giant component. We find that the system undergoes a percolation transition at certain fraction $p=p_{c}$ which is always smaller than the $p_{c}$ for randomly coupled networks with the same $P(k)$. We also find that the system undergoes a first order transition at $p_{c}>0$ if $P(k)$ has a finite second moment. For the case of scale free networks with $2<\lambda \leq 3$, the transition becomes a second order transition. Moreover, if $\lambda<3$ we find $p_{c}=0$ as in percolation of a single network. For $\lambda=3$ we find an exact analytical expression for $p_{c}>0$. Finally, we find that the robustness of CCN increases with the broadness of their degree distribution.
\end{abstract}




\section{INTRODUCTION}

The robustness of interdependent networks has been recently studied by Buldyrev et al.

[1] within the framework of the mutual percolation model. They found that two randomly connected networks with arbitrary degree distributions randomly coupled by bidirectional dependency links completely disintegrate via a cascade of failures if the fraction $p$ of the nodes which survive the initial attack is less than some critical value $p=p_{c}>0$. Moreover, the transition at $p_{c}$ is of the first order type, i.e. the fraction of the functional nodes $\mu(p)$ which survive after the cascade of failures has a step discontinuity at $p=p_{c}$ changing from $\mu_{c}=\mu\left(p_{c}\right)>0$ for $p=p_{c}$ to zero for $p<p_{c}$. This behavior was observed even for scale free (SF) networks with a power law degree distribution $P(k) \sim k^{-\lambda}$ with $2<\lambda \leq 3$. The explanation of this behavior is based on the fact that in that model the nodes with large degree (hubs) in one network may depend on the nodes with small degree in another network. The nodes with small degree can be isolated from a giant component in one network by removal of a small fraction of nodes and thus cause the malfunction of the hubs in the other network. In real world interacting networks, the hubs in one network are more likely to depend on the hubs of another networks. This can significantly enhance the robustness of the interdependent networks. In general, the correlations among the degrees of the mutually dependent nodes can be described by a matrix $P\left(k_{1} \mid k_{2}\right)$ which specifies the conditional probabilities to find a node with degree $k_{1}$ in one network, provided it depends on a node with degree $k_{2}$ in another network. This matrix can be quite complex and may depend on many parameters. For each parameter set the model can be readily studied by computer simulations, but in order to get general understanding of the correlation effects it is desirable to solve the problem analytically at least in some limiting cases.

In this paper we study the mutual percolation problem in the case of the strongest possible correlations, namely we studied the case in which both networks (A and B) have the same number of nodes $N$ connected by bidirectional dependency links establishing a oneto-one correspondence between the nodes of the two networks in such a way that mutually dependent nodes have the same number of connectivity links, i.e. their degrees coincide, i.e. $P\left(k_{1} \mid k_{2}\right)=1$ for $k_{1}=k_{2}$ and $P\left(k_{1} \mid k_{2}\right)=0$ otherwise. This implies that both networks have the same degree distribution $P(k)$. For brevity we will call such networks correspondently coupled networks (CCN), while we will refer to the model studied in Ref. 11] as randomly 
coupled networks (RCN). As in Ref. [1], we assume that the nodes in each network are randomly connected. We define the mutually connected clusters and the mutual giant component as in Ref. [1] and assume that only the nodes which belong to the mutual giant component remain functional.

We assume that initially a $1-p$ fraction of nodes are randomly removed due to an attack or failure and find analytically the fraction of nodes $\mu(p)$ which belong to the mutual giant component. We find that as in Ref. [1], the system undergoes a percolation transition at certain fraction $p=p_{c}$ which, however, is always smaller than the $p_{c}$ for RCN with the same degree distribution with the exception of random regular graphs [2] for which both values coincide. Moreover, we find that the system undergoes a first order transition at $p_{c}>0$ if the degree distribution has a finite second moment. For the practically imqportant case of SF networks[3-7] with $2<\lambda \leq 3$, for which the second moment diverges, the transition becomes a second-order transition. If $\lambda<3$ we find that $p_{c}=0$ as in percolation of a single network [8], while for $\lambda=3$ we find an exact analytical expression for $p_{c}>0$. The change in transition order has been also observed in interdependent networks with partial coupling [9]. We also investigate how the broadness of the degree distribution affects the robustness of CCN.

\section{GENERATING FUNCTIONS AND THE CASCADE PROCESS}

\section{A. First stage}

We will describe the stages of the cascade of failures in CCN in terms of the generating function of their degree distribution [10, 11]

$$
G(x)=\sum_{k=0}^{\infty} P(k) x^{k}
$$

and the generating function of the associated branching process [12]

$$
H(x)=\frac{G^{\prime}(x)}{G^{\prime}(1)}=\frac{1}{\langle k\rangle} \frac{d G(x)}{d x},
$$

where $\langle k\rangle \equiv G^{\prime}(1)$ is the average degree. It is known that the degree distribution $\tilde{P}(k, p)$ of a network, from which a fraction of nodes $1-p$ is randomly removed is related to the 
original distribution $P(k)$ through a binomial expansion [10]:

$$
\tilde{P}\left(k^{\prime}, p\right)=\sum_{k \geq k^{\prime}} P(k) p^{k^{\prime}}(1-p)^{k-k^{\prime}} C_{k}^{k^{\prime}}
$$

where $C_{k}^{k^{\prime}}=k ! /\left[k^{\prime} !\left(k-k^{\prime}\right) !\right]$ are binomial coefficients. Accordingly [10], the generating function of this distribution is

$$
\tilde{G}(x, p)=G(x p+1-p) .
$$

The fraction of nodes which do not belong to the giant component of a network is given by [13, 14]

$$
r=G(f)
$$

where $f$ is a smallest nonnegative root a transcendental equation

$$
f=H(f)
$$

The degree distribution of nodes which do not belong to the giant component is given by [13]

$$
P_{o}(k, f)=P(k) f^{k} / r \text {. }
$$

Accordingly the degree distribution of nodes in the giant component is given by

$$
P_{i}(k, f)=P(k)\left(1-f^{k}\right) /(1-r) .
$$

Thus the degree distribution in the giant component of a decimated network after random removal of a $1-p$ fraction of nodes is

$$
\tilde{P}_{i}\left(k^{\prime}, f, p\right)=\tilde{P}\left(k^{\prime}, p\right)\left[1-f(p)^{k^{\prime}}\right] /[1-r(p)]
$$

where

$$
r(p)=\tilde{G}(f(p), p)
$$

and $f(p)$ satisfies the transcendental equation

$$
f(p)=\tilde{H}(f(p), p) .
$$

In order to find the original degree distribution in the giant component of network $\mathrm{A}$ we must restore the links which lead to the randomly removed nodes. If a node in the decimated 
network A has a degree $k^{\prime}$ it might have any degree $k \geq k^{\prime}$ in the original network A with probability $P\left(k \mid k^{\prime}\right)$ given by the Bayes' formula:

$$
P\left(k \mid k^{\prime}\right)=P(k) C_{k}^{k^{\prime}} p^{k^{\prime}}(1-p)^{k-k^{\prime}} / \tilde{P}(k, p)
$$

Thus the total probability that a node in the giant component has a degree $k$ is

$$
P_{1}(k)=\sum_{k^{\prime} \leq k} P(k) C_{k}^{k^{\prime}} p^{k^{\prime}}(1-p)^{k-k^{\prime}} \frac{\tilde{P}_{i}\left(k^{\prime}, f, p\right)}{\tilde{P}\left(k^{\prime}, p\right)},
$$

or using Eq. (91)

$$
P_{1}(k)=\sum_{k^{\prime} \leq k} P(k) C_{k}^{k^{\prime}} p^{k^{\prime}}(1-p)^{k-k^{\prime}} \frac{1-f(p)^{k^{\prime}}}{1-r(p)}=P(k) \frac{1-(f(p) p+1-p)^{k}}{1-r(p)} .
$$

The generating function of this degree distribution is

$$
G_{1}(x)=\frac{G(x)-G\left(x t_{1}\right)}{1-G\left(t_{1}\right)}
$$

where $t_{1}=f_{1} p+1-p$ and $f_{1}=f(p)$. The fraction of nodes in the giant component of the decimated network $\mathrm{A}$ is $1-r_{1}$, where $r_{1}=G\left(t_{1}\right)$, Because the decimated network has $N p$ nodes, the size of the giant component $\mathrm{A}_{1}$ of network $\mathrm{A}$ after random removal of $(1-p)$ nodes is $N_{1}=N p\left(1-r_{1}\right)$.

\section{B. Second stage}

We assume that only nodes which belong to $\mathrm{A}_{1}$ are functional; thus after the first stage of the cascades of failures, only $p\left(1-r_{1}\right)<p$ fraction of nodes in network B remain functional. Thus we expect further disintegration of network B at the second stage of the cascade and its giant component $\mathrm{B}_{2}$ will be even smaller than $\mathrm{A}_{1}$. We define a set of nodes $\mathrm{B}_{1}=D\left(\mathrm{~A}_{1}\right)$ by

projecting $\mathrm{A}_{1}$ onto network $\mathrm{B}$ using the one-to-one correspondence $D$ between the nodes of networks A and B established by dependency links. Since the degree of each node in network $\mathrm{B}$ is the same as the degree of its dependent node in network $\mathrm{A}$, the giant component $\mathrm{A}_{1}$ of network A obtained at the first stage of the cascade has the same degree distribution as the set $\mathrm{B}_{1}$ in network $\mathrm{B}$. Moreover, from the point of view of network $\mathrm{B}$ the nodes in $\mathrm{B}_{1}$ are randomly selected and randomly connected. Thus, to compute $\mathrm{B}_{2}$ we can use the same approach used at the first stage, but applied to the new network $B_{1}$ with the new degree distribution given by Eq. (14). The only problem is that many of the links outgoing 
from network $B_{1}$ are ending at the nodes which do not belong to network $B_{1}$ and thus for computation of $\mathrm{B}_{2}$ these links must be removed. The probability $p_{1}$ of a random link originating in network $\mathrm{B}_{1}$ to end up in $\mathrm{B}_{1}$ is equal to the ratio of the number of links originating in network $\mathrm{B}_{1}$ :

$$
L_{1}=N_{1} \sum k P_{1}(k)=p N\langle k\rangle\left(1-G^{\prime}\left(t_{1}\right) t_{1} /\langle k\rangle\right)
$$

to the total number of links $N\langle k\rangle$. Therefore,

$$
p_{1}=\frac{L_{1}}{N\langle k\rangle}=p\left(1-s_{1}\right)
$$

where

$$
s_{1}=t_{1} G^{\prime}\left(t_{1}\right) /\langle k\rangle \text {. }
$$

Accordingly, the degree distribution of links connecting the nodes of network $\mathrm{B}_{1}$ is

$$
\tilde{P}_{1}\left(k^{\prime}, p\right)=\sum_{k \geq k^{\prime}} P_{1}(k) p_{1}^{k^{\prime}}\left(1-p_{1}\right)^{k-k^{\prime}} C_{k}^{k^{\prime}}
$$

and the generating function of this distribution is

$$
\tilde{G}_{1}\left(x, p_{1}\right)=\left[G\left(x p_{1}+1-p_{1}\right)-G\left(t_{1}\left(x p_{1}+1-p_{1}\right)\right)\right] /\left(1-r_{1}\right) .
$$

Thus the size $N_{2}$ of the giant component $\mathrm{B}_{2}$ is $N_{2}=p\left(1-r_{1}\right)\left(1-r_{2}\right) N$, where $r_{2}=\tilde{G}_{1}\left(f_{2}, p_{1}\right)$ and $f_{2}=\tilde{H}_{1}\left(f_{2}, p_{1}\right)$. Introducing a new notation

$$
t_{2} \equiv f_{2} p_{1}+1-p_{1}
$$

and taking into account Eq. (20) we see that

$$
f_{2}=\frac{G^{\prime}\left(t_{1}\right)-G^{\prime}\left(t_{1} t_{2}\right) t_{1}}{\langle k\rangle\left(1-s_{1}\right)}
$$

and $N_{2}=p\left(1-r_{1}\right)\left[1-\left(G\left(t_{2}\right)-G\left(t_{2} t_{1}\right)\right) /\left(1-r_{1}\right)\right]$. Using that $r_{1}=G\left(t_{1}\right)$, we get

$$
N_{2}=p\left[1-G\left(t_{1}\right)-G\left(t_{2}\right)+G\left(t_{1} t_{2}\right)\right] N
$$

We can compute the original degree distribution $P_{2}(k)$ in $\mathrm{B}_{2}$ using the Bayes' formula the same way as we obtained the distribution $P_{1}(k)$

$$
P_{2}(k)=P(k) \frac{\left(1-t_{1}^{k}\right)\left(1-t_{2}^{k}\right)}{1-G\left(t_{1}\right)-G\left(t_{2}\right)+G\left(t_{1} t_{2}\right)} .
$$




\section{Third stage}

On the third stage of the cascade we will compute the giant component $\mathrm{A}_{3}$ of network A which is the result of further disintegration of $A_{1}$ because the nodes in $A_{1}$ which do not belong to $\mathrm{B}_{2}$ failed at the second stage. We can again apply the same technique, with the only difference that now $\mathrm{A}_{2}=D\left(\mathrm{~B}_{2}\right)$ is not a random subset of nodes of $\mathrm{A}$ but they are taken out of its connected giant component $\mathrm{A}_{1}$. Accordingly, we must find an effective degree distribution and the effective size of a network which would reproduce $\mathrm{A}_{2}$ by random selection of nodes out of this original network. Since the degree distribution of nodes in $A_{1}$ is

$$
P_{1}(k)=P(k) \frac{1-t_{1}^{k}}{1-G\left(r_{1}\right)}
$$

and the degree distribution in $\mathrm{A}_{2}$ is

$$
P_{2}(k)=P(k) \frac{\left(1-t_{2}^{k}\right)\left(1-t_{1}^{k}\right)}{\left(1-r_{2}\right)\left(1-r_{1}\right)},
$$

the selection of $\mathrm{A}_{2}$ out of $\mathrm{A}_{1}$ has the same effect as random selection of a fraction $p\left(1-r_{1}\right)$

out of the entire network with degree distribution $P_{2}^{*}(k)=P(k)\left(1-t_{2}^{k}\right) /\left(1-r_{2}\right)$. Now we can see that the problem of the third stage is completely equivalent to the second stage with $t_{1}$ replaced by $t_{2}, f_{1}$ replaced by $f_{2}, r_{1}$ replaced by $r_{2}, t_{2}$ replaced by $t_{3}, f_{2}$ replaced by $f_{3}$, and $r_{2}$ replaced by $r_{3}$.

\section{Recursive relations}

Generalizing, for stage $i$ we arrive to a recursive relation between $t_{i}$ and $t_{i+1}$. Namely, once we know $t_{i}$ we can find $t_{i+1}$, as well as the size of the giant component at the stage $i+1$

$$
N_{i+1}=p\left[1-G\left(t_{i}\right)-G\left(t_{i+1}\right)+G\left(t_{i} t_{i+1}\right)\right] N
$$

and the degree distribution of the nodes inside this giant component

$$
P_{i+1}(k)=P(k) \frac{\left(1-t_{i+1}^{k}\right)\left(1-t_{i}^{k}\right)}{1-G\left(t_{i}\right)-G\left(t_{i+1}\right)+G\left(t_{i} t_{i+1}\right)}
$$

In order to find $t_{i+1}$ from $t_{i}$ we repeat the steps used deriving $t_{2}$ from $t_{1}$ by first introducing

$$
s_{i}=t_{i} G^{\prime}\left(t_{i}\right) /\langle k\rangle,
$$


and

$$
p_{i}=p\left(1-s_{i}\right)
$$

in analogy to Eqs. (29) and (17). Then

$$
t_{i+1} \equiv f_{i+1} p_{i}+1-p_{i}
$$

where $f_{i+1}$ satisfies a transcendental equation analogous to Eq. (22)

$$
f_{i+1}=\frac{G^{\prime}\left(t_{i}\right)-G^{\prime}\left(t_{i} t_{i+1}\right) t_{i}}{\langle k\rangle\left(1-s_{i}\right)}
$$

Excluding $f_{i+1}$ and $s_{i}$ from Eq. (321) we find that $t_{i+1}$ is given by the smallest non-negative root of the equation

$$
t_{i+1}=(1-p)+\frac{p}{\langle k\rangle}\left[G^{\prime}\left(t_{i}\right) t_{i}+G^{\prime}\left(t_{i+1}\right)-t_{i} G^{\prime}\left(t_{i} t_{i+1}\right)\right] .
$$

To start the iterative process we must take into account the definition of $t_{1}=p f_{1}+1-p$ and Eq. (11) which is equivalent to a transcendental equation

$$
t_{1}=(1-p)+\frac{p}{\langle k\rangle} G^{\prime}\left(t_{1}\right)
$$

which is the same as Eq. (33) if we introduce $t_{0} \equiv 0$.

\section{THE MUTUAL GIANT COMPONENT AND THE PHASE TRANSITION}

The cascade of failures will stop when $t_{i+1}=t_{i}=t$ and hence the fraction of nodes in the mutual giant component $\mu=\lim _{i \rightarrow \infty} N_{i} / N$, is given by the simplified equation (27):

$$
\mu=p\left[1-2 G(t)+G\left(t^{2}\right)\right]
$$

where $t$ is the smallest non-negative root of the equation

$$
t=(1-p)+\frac{p}{\langle k\rangle}\left[(1+t) G^{\prime}(t)-t G^{\prime}\left(t^{2}\right)\right]=1-p\left[1-(1+t) H(t)+t H\left(t^{2}\right)\right] .
$$

The right hand side of Eq. (36) has zero derivative at $t=1$, if $G^{\prime \prime}(1)$ is finite. This condition is equivalent to the existence of the second moment of the degree distribution. Thus one can see [Fig. $\mathbb{1}(\mathrm{a}, \mathrm{b})]$ that for finite second moment and small enough $p$, Eq. (36) has only the trivial solution $t=1$ corresponding to $\mu=0$ and, therefore, to the complete 
disintegration of the networks. As $p$ increases, a nontrivial solution $\mu>0$ will emerge at $p=p_{c}$, at which point the right hand side of Eq. (36) will touch the straight line representing the left hand side at $t=t_{c}$; at that point the slope of both lines is equal to 1 . Since at $t=1$ the slope of the right hand side is zero, $t_{c}$ must be smaller than 1 and thus the mutual percolation transition is of the first order, where $\mu$ changes form zero (for $p<p_{c}$ ) to $\mu \geq \mu_{c}>0$ (for $p \geq p_{c}$ ). The value of $\mu_{c}$ is given by Eq. (35) computed at $t=t_{c}$.

An efficient way of finding $p_{c}$ is to solve Eq. (36) with respect to $1 / p$ :

$$
\frac{1-(1+t) H(t)+t H\left(t^{2}\right)}{1-t}=\frac{1}{p}
$$

and find the maximum of the left hand side with respect to $t$ [Fig. 2]. The left hand side of Eq. (37) is a curve which changes from $1-H(0)=1-P(1) /\langle k\rangle$ at $t=0$ to zero at $t=1$. At $t=0$ it has a positive slope $1-(P(1)+2 P(2)) /\langle k\rangle)$, so it must have an absolute maximum at $t_{c} \in(0,1)$. The equation for $t_{c}$ can be readily obtained by differentiation of Eq. (37)

$$
1-2 H\left(t_{c}\right)+H\left(t_{c}^{2}\right)-\left(1-t_{c}^{2}\right) H^{\prime}\left(t_{c}\right)+2 t_{c}^{2}(1-t+c) H^{\prime}\left(t_{c}^{2}\right)=0
$$

The value of the left hand side of Eq. (37) at $t=t_{c}$ gives $1 / p_{c}$. If the value of this maximum is less than 1 , than the networks do not have a mutual giant component at any $p$.

\section{SPECIAL CASES}

Figure 1 shows the graphical solutions of Eq. (36) for several special cases of degree distributions of CCN.

\section{A. Erdős-Rényi networks}

For Erdős-Rényi (ER) networks[2, 15] $H(t)=\exp [\langle k\rangle(t-1)]$ and the maximal value of the left hand side of Eq. (37) monotonously increases with $\langle k\rangle$. This can be readily seen by differentiating Eq. (37) with respect to $\langle k\rangle$. The maximal value reaches 1 at $\langle k\rangle=1.706526$, below which correspondently coupled ER networks disintegrate even without any initial attack or failure (Fig. 2). Note that the equivalent value of $\langle k\rangle$ for randomly coupled ER networks is $2.4554[1]$. 


\section{B. Random regular graphs}

For RR graph [Fig. 1(b)] in which all the nodes have the same degree $k=\langle k\rangle, G(t)=t^{\langle k\rangle}$ and $H(t)=t^{\langle k\rangle-1}$. Then $t$ satisfies

$$
t=(1-p)+p\left(t^{\langle k\rangle-1}+t^{\langle k\rangle}-t^{2\langle k\rangle-1}\right)
$$

and

$$
\mu=p\left(1-t^{\langle k\rangle}\right)^{2}
$$

Equations (39) and (40) can be obtained by simpler methods presented in Ref. [1] for RCN, since for the case of random regular graphs, the degrees of all the nodes in both networks coincide and therefore the $\mathrm{CCN}$ and RCN models are equivalent. Indeed from Eq. (1) of Ref. [1] it follows in a special case of coinciding degree distributions of the coupled networks that

$$
\mu=p(1-G(t))^{2}
$$

where

$$
t=1-p(1-G(t))(1-H(t)) .
$$

If $G(t)=t^{\langle k\rangle}$ and $H(t)=t^{\langle k\rangle-1}$, Eqs. (41) and (42) are equivalent to Eqs. (40) and (39), respectively.

\section{Scale free networks}

For scale free (SF) networks with $\lambda<3$ [Fig. 1(c)], the second derivative of the right hand side of Eq. (36) is infinite at $t=1$, which means that a nontrivial solution exists at any $p>0$ since in the vicinity of $t=1$ the straight line representing the left hand side of Eq. (36) is always above the curve representing the right hand side, while for $t=0$, the curve is always above the line. This means that SF CCN are as robust as a single SF network for which $p_{c}$ is always zero.

For the marginal case of $\lambda=3$ [Fig. [1(d)] $G^{\prime \prime}(t)$ diverges as $\ln (1-t)$ when $t \rightarrow 1$ and thus the left hand side of Eq. (36) has a finite slope at $t=1$. Accordingly $p=p_{c}>0$ but the nontrivial solution emerges at $t_{c}=1$, so the transition becomes of the second order. For the

case of $P(k)=\left(k_{\text {min }} / k\right)^{2}-\left(k_{\text {min }} /(k+1)\right)^{2}$ for $k \geq k_{\text {min }}=1,2, .$. and $P(k)=0$ for $k<k_{\text {min }}$ we can find $p_{c}$ analytically. Indeed, in this case $P(k)$ behaves asymptotically as $2 k_{\min }^{2} / k^{3}$. 
For $k \rightarrow \infty$ the leading term in $G^{\prime \prime}(t)$ becomes $2 k_{\text {min }}^{2} t^{k} / k$; so, $G^{\prime \prime}(t)=-2 k_{\text {min }}^{2} \ln (1-t)+c(t)$, where $c(t)$ is a continuous function. Accordingly, the slope of the right hand side of Eq. (36) at $t=1$ becomes $p 4 k_{\text {min }}^{2} \ln (2) /\langle k\rangle$, where

$$
\langle k\rangle=k_{\min }+k_{\min }^{2}\left(\frac{\pi^{2}}{6}-\sum_{k=1}^{k_{\min }} \frac{1}{k^{2}}\right) .
$$

The critical threshold is thus

$$
p_{c}=\frac{\frac{1}{k_{\min }}+\frac{\pi^{2}}{6}-\sum_{k=1}^{k_{\min }} \frac{1}{k^{2}}}{4 \ln (2)}
$$

For $k_{\text {min }}=1 p_{c}=0.59328456$ and for $k_{\text {min }}=2 p_{c}=0.32277924$.

\section{Effect of the broadness of the degree distribution}

It follows from Fig. 1 that for the same $\langle k\rangle=3, p_{c}$ of the RR networks (0.758751) is greater than the $p_{c}$ of the ER networks (0.6499451). Moreover, for SF networks with $\lambda=3$ and $k_{\text {min }}=1$, for which the average degree is $\pi^{2} / 6<3$ we have even smaller $p_{c}=0.59328456$. For SF networks with $\lambda=3,\langle k\rangle=3$ we can estimate $p_{c}=0.35$ which is much smaller than the $p_{c}$ for the narrower ER and RR degree distribution. For SF networks with $\lambda<3$, which are even broader, $p_{c}=0$ for any $\langle k\rangle$. This is in a complete agreement with the trend observed in percolation of single networks, for which the robustness increases with the broadness of the degree distribution if one keeps $\langle k\rangle$ constant, but is opposite to the trend observed in Ref. [1] for RCN.

In order to investigate the effect thoroughly, we study several classes of degree distributions for a number of values of $\langle k\rangle$. Figure 3 shows $p_{c}$ as function of $\langle k\rangle$ for RR, ER, uniform, and SF with $\lambda=3$ degree distributions. For each value of $\langle k\rangle$ the variance of $\mathrm{SF}$ degree distribution $(\infty)$ is greater than the variance of the uniform degree distribution $(\langle k\rangle(\langle k\rangle+1) / 3)$, which is greater than the variance of ER degree distribution $\langle k\rangle$ which is greater than the variance of RR degree distribution (0). Indeed, Fig. 3 shows that $p_{c}(\mathrm{SF})<p_{c}$ (uniform) $<p_{c}(\mathrm{ER})<p_{c}(\mathrm{RR})$. Thus our numerical results suggest that CCN become more robust if their degree distribution becomes broader (provided the average degree is constant). This behavior is the opposite of the behavior of RCN.

However, in general, if the measure of broadness is simply the variance of the degree distribution, our statement is incorrect. It is possible to find two distributions with the same 
variances and average degrees, which have different values of $p_{c}$. One particular example is the following two distributions $P_{1}(0)=0, P_{1}(1)=P_{1}(2)=P_{1}(3)=P_{1}(4)=P_{1}(5)=1 / 5$ and $P_{2}(0)=P_{2}(3)=0, P_{2}(1)=P_{2}(5)=1 / 6, P_{2}(2)=P_{2}(4)=1 / 3$ which have $p_{c}$ respectively 0.683099 and 0.683657 .

\section{GENERAL IMPLICATIONS ON THE NETWORK ROBUSTNESS}

Finally, we will compare the robustness of CCN and RCN with the same degree distributions. We will show that $(i)$ the value of $p_{c}$ for $\mathrm{CCN}$ is always (except for RR networks) smaller than the $p_{c}$ for $\mathrm{RCN}$ and $(i i)$ for the same $p$, the value of the mutual giant component for CCN is always (except for RR networks) larger than for RCN.

Eq. (42) for the randomly coupled networks can be rewritten as

$$
\frac{[1-H(t)][1-G(t)]}{1-t}=\frac{1}{p}
$$

The critical value of $p_{c}$ for randomly coupled networks can be determined as the inverse maximal value of the left hand side of Eq. (45). Our proposition $(i)$ is an obvious corollary of the following proposition (iii): for any $t \in[0,1]$ the left hand side of Eq. (37) is greater or equal than the left hand side of Eq. (45) (Fig,2). Subtracting Eq. (45) from Eq. (37) and applying relation (2) between $G(t)$ and $H(t)$ we see that the inequality stated in proposition (iii) is equivalent to

$$
t G^{\prime}\left(t^{2}\right)-t G^{\prime}(t)+G(t) G^{\prime}(1)-G(t) G^{\prime}(t) \geq 0
$$

We will prove Eq. (46) using mathematical induction. We see that for RR graphs for which the degree of every node is equal to $m$, i.e. $P(m)=1$, Eq. (46) is satisfied as an equality. Assuming that it is satisfied for any degree distribution such that $P(k)=0$ for $k<m$ and $k>n \geq m$, we will show that it is also satisfied for the degree distribution $\tilde{P}(k)=(1-b) P(k)$ for any $k$ except for $k=n+1$, for which $\tilde{P}(n+1)=b>0$. The generating function for this new distribution is obviously $\tilde{G}=(1-b) G+b t^{n+1}$. After elementary algebra we can see that

$$
\begin{gathered}
t \tilde{G}^{\prime}\left(t^{2}\right)-t \tilde{G}^{\prime}(t)+\tilde{G}(t) \tilde{G}^{\prime}(1)-\tilde{G}(t) \tilde{G}^{\prime}(t)= \\
(1-b)\left[t G^{\prime}\left(t^{2}\right)-t G^{\prime}(t)+G(t) G^{\prime}(1)-G(t) G^{\prime}(t)\right]+ \\
b(1-b)\left(G(t)-t^{n+1}\right)\left[\left(n+1-G^{\prime}(1)\right)\left(1-t^{n}\right)+G^{\prime}(t)-G^{\prime}(1) t^{n}\right],
\end{gathered}
$$


which proves Eq. (46) for $\tilde{G}$ provided it is true for $G$, if we take into account the obvious inequalities $n+1>G^{\prime}(1), 1 \geq t^{n}, G(t) \geq t^{n+1}$ and $G^{\prime}(t) \geq G^{\prime}(1) t^{n}$ for any $t \in[0,1]$. This concludes the proof of propositions $($ iii $)$ and $(i)$. Note that the equality sign in the above inequalities and hence in inequality (46) is realized only for $t=1$ and $t=0$ (if $P(0)=0)$. Hence proposition $(i)$ always implies rigorous inequality except for the case of RR graphs.

To prove the proposition ( $i i)$ we first notice that the smallest positive root of Eq. (37), $t_{1}$, is always smaller than the smallest positive root $t_{2}$ of Eq. (45). This a is direct consequence of proposition (iii). Also we notice that the right hand side of Eq. (35) is a monotonously decreasing function of $t$. This can be shown by differentiation and comparing the terms of $G^{\prime}(t)$ and $t G^{\prime}\left(t^{2}\right)$ corresponding to the same $P(k)$, namely $k P(k) t^{k-1} \geq k P(k) t^{2 k-1}$. Thus $\mu\left(t_{1}\right)>\mu\left(t_{2}\right)$. Finally, we state proposition $(i v)$ : for the same value of $t$, the right hand side of Eq. (35), $\mu(t)$, is greater or equal than the right hand side of Eq. (41), $\mu_{r}(t)$. One can prove this proposition using the same induction method we used to prove proposition (iii). Combining these two results $\mu\left(t_{1}\right)>\mu\left(t_{2}\right) \geq \mu_{r}\left(t_{2}\right)$, which concludes the proof of proposition $(i i)$.

Thus CCN are more robust than RCN with the same $P(k)$, but are still prone to cascade failures and, then, to first order disintegration (only if $G^{\prime \prime}(1)<\infty$ ) as in case of randomly coupled networks.

\section{SUMMARY}

In this work we have studied the problem of failure of $\mathrm{CCN}$, i.e. coupled networks with coinciding degrees of mutually dependent nodes. We derive new recursive equations [Eqs. (33) and (27)] describing the cascade of failures that are different from the analogous equations for RCN studied in Ref. [1]. We also find equations for the size of the mutual giant component [Eqs.(35) and (36)], as well as the efficient way of finding the critical fraction of nodes $p=p_{c}$ which must survive the initial random failure for the mutual giant component not to vanish, by finding the maximum of Eq. (37).

We show that if the second moment of the degree distribution is finite, CCN disintegrate in a cascade of failures via a first order transition at which the mutual giant component suddenly drops from a positive fraction above $p_{c}>0$ to zero below $p_{c}$. This behavior is analogous to the behavior of RCN, with the only difference that RCN disintegrate via a first 
order transition even when the second moment of their degree distribution diverges.

Moreover, we show that $\mathrm{CCN}$ are always more robust than $\mathrm{RCN}$ with the same degree distribution. In particular, we show that scale free CCN with $\lambda<3$ disintegrate via a second order phase transition in the same way as non-interacting networks and thus are very resilient against random failure. Namely, the mutual giant component for these networks exists at any $p>0$, but becomes infinitely small as $p \rightarrow 0$. Finally CCN become more robust if their degree distribution becomes broader (provided the average degree is constant). This behavior is the opposite of the behavior of RCN.

All our analytical predictions are confirmed by simulations of coupled networks with large number of nodes $\left(N \geq 10^{6}\right)$.

Based on our findings we conjecture that coupled networks with any positively correlated degrees of mutually dependent nodes (and not just the present case of fully coincidental

degrees) are more robust that their randomly coupled counterparts studied in Ref. [1] This can be attributed to the fact that the correlation between the degrees of nodes suppresses (or attenuates) the phenomenon of hubs becoming more vulnerable by being dependent on low degree nodes in a coupled network.

\section{ACKNOWLEDGMENTS}

We wish to thank DTRA for financial support and Dr. Robin Burk for encouraging discussions. We acknowledge the partial support of this research through the Dr. Bernard W. Gamson Computational Science Center at Yeshiva College.

[1] S.V. Buldyrev, R. Parshani, G. Paul, H. E. Stanley, and S. Havlin, Nature (London) 464, 1025 (2010).

[2] B. Bollobás, Random Graphs (Academic, London, 1985).

[3] A.-L. Barabási and R. Albert, Science 286, 509 (1999).

[4] R. Albert and A.-L.Barabási, Rev. Mod. Phys. 74, 47(2002).

[5] R. Pastor-Satorras and A. Vespignani, Evolution and Structure of the Internet: A Statistical Physics Approach (Cambridge University Press, 2006). 
[6] S. N. Dorogovtsev and J. F. F. Mendes, Evolution of Networks: from Biological nets to the Internet and $W W W($ Oxford University Press, New York, 2003).

[7] R. Cohen and S. Havlin, Phys. Rev. Lett. 90, 058701 (2003).

[8] R. Cohen, K. Erez, D. ben-Avraham, and S. Havlin, Phys. Rev. Lett. 85, 4626 (2000); Ibid. 86, 3682 (2001).

[9] R. Parshani, S. V. Buldyrev, and S. Havlin, Phys. Rev. Lett. 105048701 (2010).

[10] M. E. J. Newman, Phys. Rev E 66, 016128 (2002).

[11] M. E. J. Newman et al., Phys. Rev. E 64, 026118 (2001).

[12] T. E. Harris, Ann. Math. Statist. 41, 474 (1948); The Theory of Branching Processes (Springer-Verlag, Berlin, 1963).

[13] J. Shao, S. V. Buldyrev, L. A. Braunstein, S. Havlin, and H. E. Stanley, Phys. Rev. E 80, 036105 (2009).

[14] J. Shao, S. V. Buldyrev, R. Cohen, M. Kitsak, S. Havlin and H. E. Stanley, Europhys. Lett. 84, 48004 (2008).

[15] P. Erdős and A. Rényi, Publ. Math. 6, 290 (1959); Publ. Math. Inst. Hung. Acad. Sci. 5, 17 (1960). 

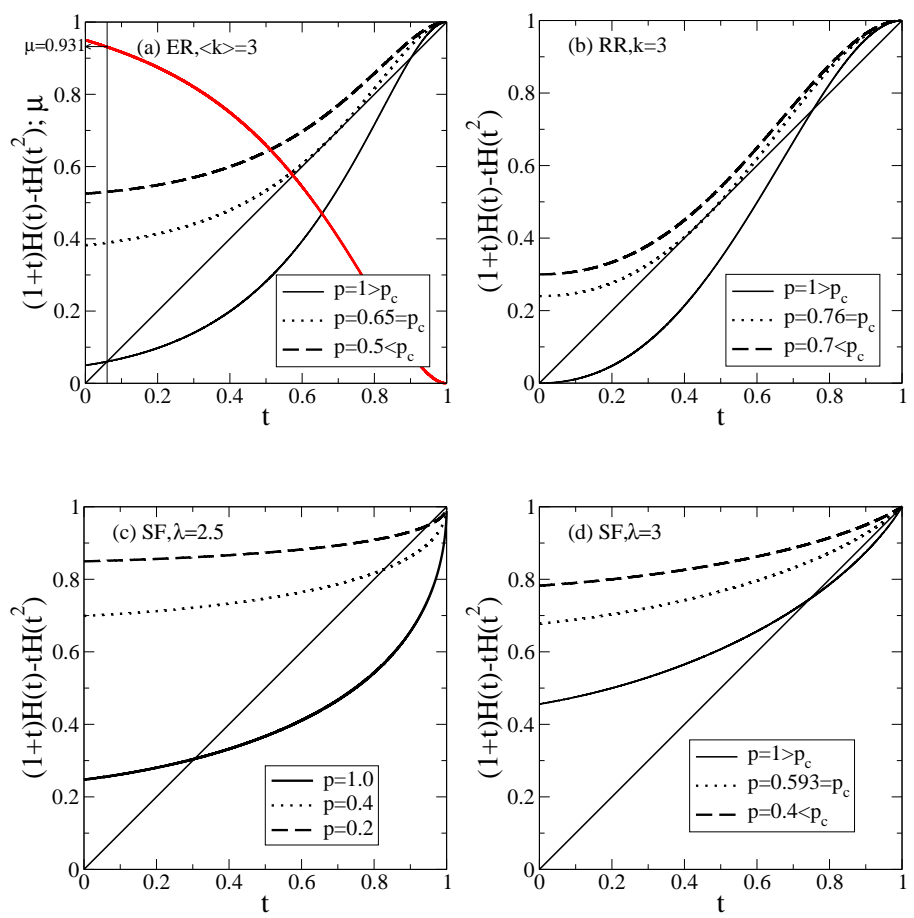

FIG. 1: Graphical solution of Eq. (36) for various special cases of CCN. (a) ER networks with average degree $\langle k\rangle=3$. One can see that the black curves representing the right hand side of Eq. (36) for different $p$ have zero slopes at $t=1$. The relevant solutions for $t$ are given by the lower intersection points of these curves and a straight line $y=t$ representing the left hand side of Eq. (36). For $p=1$, this solution $t=0.0602$ is indicated by a vertical straight line. The intersection of this vertical line with the plot of Eq. (35) (red curve) gives the mutual giant component $\mu=0.931$. The critical $p=p_{c}=0.6499451$ corresponds to sudden disappearance of the nontrivial solution. (b) RR networks with $k=3$. Note that for $p=1$ the nontrivial solution is $t=0$ which means that $\mu=1$. The value of $p_{c}=0.758751$ is grater than the $p_{c}$ for ER networks with the same average degree shown in panel (a). (c) Analogous plot for scale free networks with $\lambda=2.5$. It shows that the slope of the curves is infinite for $t \rightarrow 1$. One can see that in this case the nontrivial solution exists for any $p>0$. However as $p \rightarrow 0$, the nontrivial solution $t \rightarrow 1$, and, accordingly, $\mu \rightarrow 0$ indicating the second order transition at $p=p_{c}=0$. (d) The marginal case of $\lambda=3$. The slopes of the curves for $t \rightarrow 1$ are finite. This means that there is a critical $p=p_{c}>0$ at which the slope of the curve becomes equal to 1 at $t \rightarrow 1$. For the displayed case of $k_{\min }=1$, Eq. (44) yields $p_{c}=0.59328456$. The nontrivial solution smoothly approaches 1 as $p \rightarrow p_{c}$. This again implies $\mu \rightarrow 0$ (second order transition). 


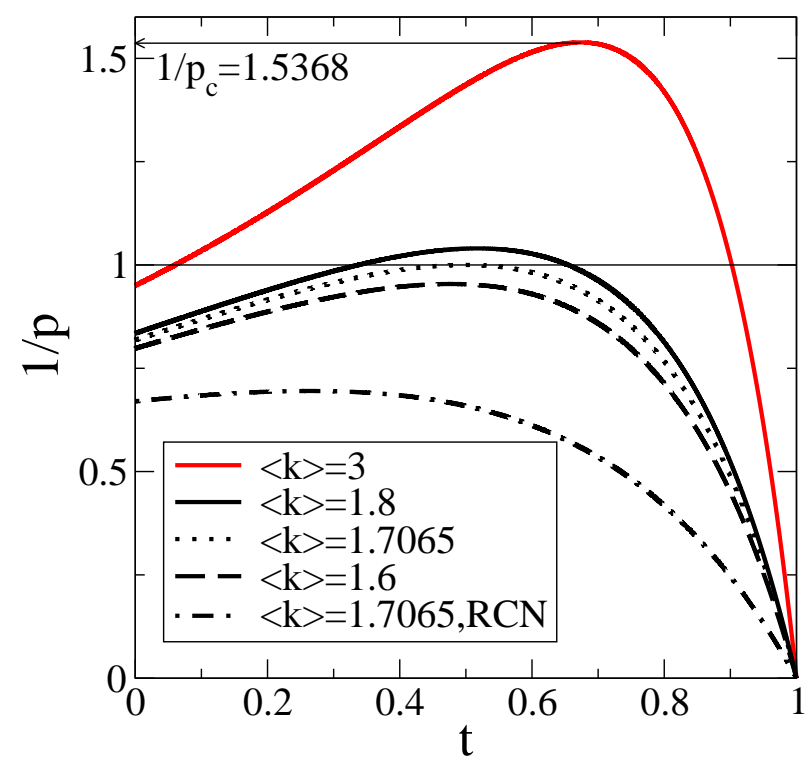

FIG. 2: Graphical solution of Eq. (37) for ER networks with different degree $<k>$ illustrating the method of finding $p_{c}$. The red curve corresponds to $\langle k\rangle=3$ studied in Fig.1(a). As $<k>$ decreases below 1.706, the nontrivial solution corresponding to $p \leq 1$ disappears. We also show the behavior of the analogous equation (45) for $\langle k\rangle=1.706$ for RCN. In agreement with proposition (iii) this curve is always below the curve with the same average degree for networks with coinciding degrees studied here. 


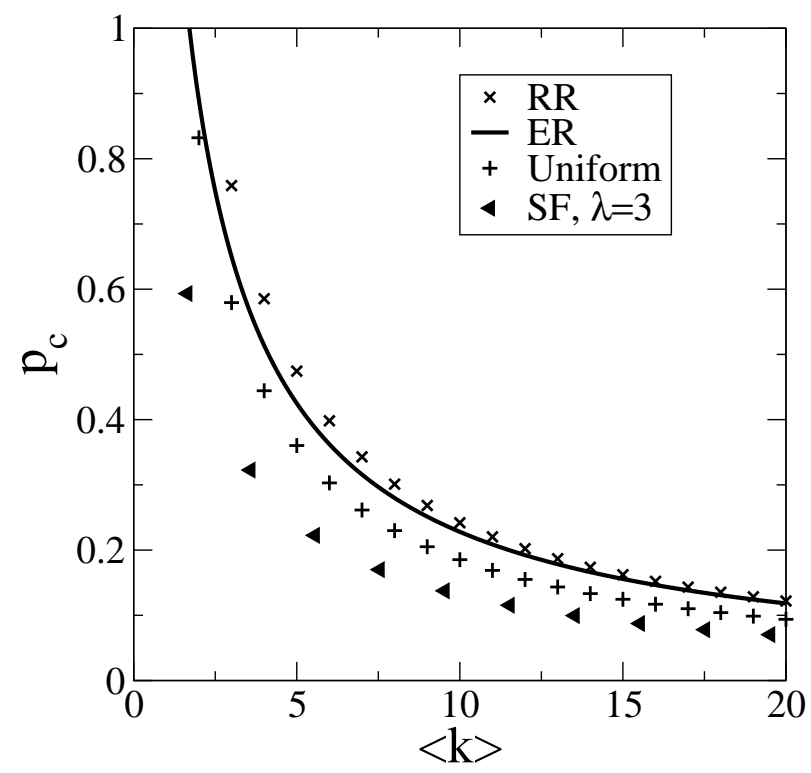

FIG. 3: The values of $p_{c}$ versus $\langle k\rangle$ for several degree distributions of increasing broadness, namely RR, ER, uniform and SF with $\lambda=3$. We define the uniform distribution as follows: $P(k)=1 /(2\langle k\rangle+1)$ for $k=0,1, \ldots, 2\langle k\rangle$ and $P(k)=0$ for $k>2\langle k\rangle$. For SF distribution we use Eqs. (43) and (44), while for other distributions we numerically solve Eq. (38) and use Eq. (37) to find $p_{c}$. One can see that $p_{c}$ decreases ( and hence the robustness increases) with the broadness. 